\title{
Oxyanion adsorption structures, thermodynamics and kinetics: Review of density functional theory results in comparison to experimental observations
}

\author{
NADINE KABENGI ${ }^{1}$ AND JAMES D KUBICKI ${ }^{2}$ \\ ${ }^{1}$ Georgia State University \\ ${ }^{2}$ University of Texas at El Paso \\ Presenting Author: jdkubicki@utep.edu
}

Molecular-level information on oxyanion adsorption chemistry is useful for understanding and predicting macroscale behavior. This talk will integrate adsorption isotherm, thermodynamic, spectroscopic and adsorption/desorption rate data with quantum mechanical density functional theory (DFT) calculations to demonstrate the complementary nature of these observations and simulations. By examining a range of oxyanions such as carbonate, arsenate, phosphate, silicate, chromate and sulfate, we can determine general principles of bonding that control the energetics of adsorption and the kinetics of desorption. Substrates of interest include rutile as a well-constrained model system and goethite, hematite and ferrihydrite as common reactive surfaces in the environment. In addition to modeling various surfaces of minerals, we explore reactions on ferrihydrite nanoparticles that include defects such as steps and edges representing more highly reactive sites. By comparing DFT models with observed IR spectra, EXAFS data, measured enthalpies of adsorption and sorption activation energies, we simultaneously test the accuracy of the models and fill in missing information on surface chemistry that is unavailable from direct observations. 